\title{
Los desafíos de la Autogestión Hospitalaria
}

\author{
OSVALDO ARTAZA B. ${ }^{1}$
}

1. Director Hospital Luis Calvo Mackenna; Docente Instituto de Administración en Salud (IAS) Facultad de Economía y Negocios Universidad de Chile; Secretario Ejecutivo de la Sociedad Chilena de Administradores en Atención Médica y Hospitalaria; Ex Ministro de Salud.

Chile ha alcanzado indicadores sanitarios cercanos a los de países desarrollados. Esto se logró fundamentalmente debido a que las condiciones generales del país como el nivel de vida y las condiciones sanitarias y educacionales han mejorado substancialmente en las últimas décadas junto a la contribución de un sistema de salud de amplia cobertura.

El sistema sanitario enfrenta nuevos desafíos, resultado del cambio de estructura de la población (transición demográfica avanzada); cambio de perfil epidemiológico, que se manifiesta en las principales causas de muerte (cardiovasculares, cáncer, traumatismos); aumento de expectativas y exigencias de calidad de la atención por parte de la población usuaria del sistema; introducción de tecnología y procedimientos de alto costo, y cambios profundos en aspectos políticos y económicos de gran impacto en el sector, entre otros ${ }^{1}$.

Por dichas razones desde los años noventa se vive, particularmente con relación a la red hospitalaria pública, una percepción de grave crisis, especialmente de expectativas, entre la demanda creciente de la población y una capacidad de oferta del sector deteriorada por la falta de inversión vivida durante la década anterior. Es así, que a inicios de esos años, se colocó gran énfasis en la recuperación de la infraestructura, tecnología y recurso humano del sector público, junto a aumentar cobertura y resolutividad de la atención primaria municipal. Como en muchos de los países latino americanos, los hospitales concentran una altísima proporción del gasto público en salud, su nivel de productividad era bajo y las quejas de sus usuarios guardaban relación con dificultades de acceso (las listas de espera) y con trato inadecuado, lo que generó un fuerte debate sobre el nivel de productividad y eficiencia del sistema hospitalario ${ }^{2}$.

Lo anterior explica los esfuerzos desarrollados en mejorar la eficiencia y la calidad en el sistema público hospitalario, como son diversas fórmulas para incentivar productividad y el diseño de mecanismos de pago asociados a prestaciones efectuadas y luego a resolución de diagnósticos bajo la denominación de "programas valorados"3,4; esfuerzos por capacitar en gestión; programas de introducción de metodologías de mejora continua de la calidad, y programas de excelencia hospitalaria, entre otras estrategias sostenidas en los últimos años 5 .

Una vez solucionados los aspectos más críticos de infraestructura y obsolescencia tecnológica de la red hospitalaria, el problema central del sector previo a la reforma podía resumirse, entre otras, en una incapacidad de los gestores hospitalarios para adaptar la oferta hospitalaria a las nuevas necesidades de salud de la población, y para generar políticas efectivas de recursos humanos, iniciándose un lento y paulati-

Correspondencia a:

Osvaldo Artaza B.

E-mail: oartaza@calvomackenna.cl 
no cambio de cultura organizacional en el sistema público ${ }^{6-10}$, esfuerzos que han sido esenciales en posibilitar el actual proceso de reforma.

La reforma a la salud consta de varios cuerpos legales aprobados entre los años 2003 y 2005, los que guardan relación con una mayor capacidad regulatoria del Estado a los actores privados; un plan de salud obligatorio y de acceso universal (garantías en salud, GES) que cubre el $70 \%$ de la carga de enfermedad de la población (conocido como plan "AUGE" o Acceso Universal con Garantías Explícitas); separación de funciones en las acciones de rectoría; financiamiento y prestación de servicios, y en la implementación de la "autogestión en red" para los hospitales. El concepto de autogestión en implementación implica dependencia de los hospitales en materia de la cartera de servicios (oferta sanitaria que es definida por la red pública), y una mayor desconcentración e independencia en materias de gestión, todo esto en un contexto de redes de atención estructuradas en torno a un Modelo de Atención Integral en Salud $^{11}$, el cual fue definido por el Ministerio de Salud como el conjunto de acciones organizadas del sistema orientadas a promover la calidad de vida de las personas, consideradas en su integralidad tanto individual como social, y que se caracteriza por estar centrado en el usuario; por el énfasis en lo promocional y preventivo; por su enfoque familiar y comunitario y por garantizar la integralidad y continuidad de los cuidados junto a promover la resolución ambulatoria y participativa de las necesidades de salud.

La Ley de Autoridad Sanitaria ${ }^{12}$, entrega nuevas facultades regulatorias al MINSAL, redistribuye las funciones regulatorias de los Servicios de Salud traspasándolas a las Secretarías Regionales Ministeriales (SEREMIS), reorganiza el MINSAL en dos Subsecretarías ${ }^{13}$, crea la Superintendencia de Salud, establece un nuevo rol para los Servicios de Salud, y crea la figura de los establecimientos autogestionados en red EAR.

La creación de los hospitales autogestionados tiene a lo menos tres importantes consecuencias organizacionales: primero, el hospital autogestionado siguiendo las metas asistenciales trazadas por su red, debe con sus equipos clínicos construir un plan de actividades y el plan de inversiones que éstas requieren, las cuales fundamentarán la propuesta de presupuesto que el establecimiento negociará, debiéndose aprobar por los ministerios de Salud y Hacienda antes del 15 de diciembre de cada año. Los montos no aprobados deben ser explícitamente señalados como disminución de su plan de actividades de forma de asegurar el equilibrio financiero de estos establecimientos; segundo, tienen la posibilidad, respetando su marco presupuestario, de contratar personas naturales y/o jurídicas aún en funciones propias, hasta un tope de un $20 \%$ de su presupuesto, de forma de asegurar el recurso humano que requiere su plan de actividades; tercero, tienen la facultad de darse su propia organización interna, así como proponer modificaciones a las glosas presupuestarias, celebrar contratos y convenios -en particular con FONASA, seguros privados y centros formadores-, dentro de otras nuevas facultades de administración.

Se han definido igualmente nuevas facultades que tienen como objeto modelar el desarrollo del sistema público prestador en torno al concepto de redes integradas de atención; alinear al sistema público prestador al proceso de cambio de modelo de atención; generar las condiciones organizacionales al sistema prestador para ajustar su oferta a las nuevas necesidades de salud, expresadas a través del régimen de garantías en salud (GES) y de las demás políticas ministeriales en el ámbito de la prevención, fomento, reparación y rehabilitación de problemas de salud de la población; lograr hospitales más amigables con el usuario, y mejorar las condiciones generales para aumentar la efectividad, calidad y eficiencia de los establecimientos a través de una gestión clínica de excelencia.

En el logro de estos objetivos son esenciales los siguientes aspectos: 1) que los establecimientos alcancen los niveles exigidos de "madurez" organizacional, a través del logro de estándares básicos en gestión clínica y descentralización interna; gestión de su recurso humano; gestión financiera y logística; políticas propias de investigación y docencia; trabajo coordinado en redes; participación ciudadana, etcétera, que permitieran organizaciones capaces de soste- 
ner eficientemente procesos asistenciales de calidad alineados con la priorización de carácter nacional y de la red territorial; 2) que los EAR exploren y utilicen sus nuevas facultades de modo de acelerar los procesos de implantación de cambio de modelo y ajuste de oferta; 3) que se genere la capacidad de liderazgo en los establecimientos para conducir eficazmente los procesos de cambio organizacional involucrados; 4) que las Direcciones de Servicio efectivamente ajusten su rol a "gestionar la red", lo que implica que deben hacerse cargo de definir la cartera de servicios de los establecimientos y generar las tensiones para el ajuste de oferta y la implantación del cambio de modelo, y 5) que existan los incentivos financieros para que las anteriores se den. Esto último implica buenos contratos -confiables y claros- que den soporte a una actividad asistencial en un contexto sostenible (equilibrio financiero, recursos humanos, tecnológicos y de infraestructura adecuados).

Luego de la aprobación de la ley de Autoridad Sanitaria, se firma el decreto 38 del año 2005 que establece el Reglamento de los EAR, y define un exigente instrumento de evaluación para acceder y mantenerse en la categoría de "autogestionados en red" -instrumento basado en la ley y en el reglamento- y una política de gestión hospitalaria ${ }^{14}$. El instrumento de evaluación -sobre ámbitos asistenciales y administrativos- fue consensuado con el Ministerio de Hacienda y está en aplicación.

La política -o modelo de gestión-, en lo esencial señala la misión de los establecimientos y los elementos estratégicos de las organizaciones hospitalarias en el ámbito de liderazgo y desarrollo organizacional, gestión de recursos humanos, planeamiento estratégico, gestión clínica, y en la introducción de nuevas herramientas tales como atención progresiva; gestión del cuidado; ambulatorización de los procesos asistenciales; control social y participación usuaria; mejora continua de la calidad; seguridad del paciente y costo efectividad de los procesos y de la introducción de tecnologías.

El modelo, en todo armónico con el sistema de evaluación EAR actualmente en vigencia, da cuenta de que el ministerio siempre ha entendido a la autogestión como un instrumento -no un objeto en si mismo- de la reforma. Instrumento al servicio, no del mero incremento de facultades y atribuciones a las direcciones de los establecimientos, sino que de herramientas de gestión que deben traducirse en mayores competencias para los equipos clínicos, para mejorar el servicio a los usuarios. Es así, que se inició un proceso que debiera culminar con que los principales hospitales del país estén en condiciones (según el instrumento de acreditación) de acceder a dicha categoría antes del año 2009.

El proceso no ha sido fácil, completándose sólo 11 (de 59) establecimientos para el 2008, destacando como elementos críticos: 1) que la mayoría de los hospitales no ha alcanzado los estándares requeridos, algunos (los menos) exclusivamente por inflexibilidad de los instrumentos, y los otros (desafortunadamente la mayoría) por aún importantes insuficiencias organizacionales. Hay establecimientos, claramente identificados por el Ministerio, que están potencialmente cerca de acceder pero requerirán de un enorme gasto de energía en acelerar sus procesos de mejora dada la cercanía del año 2009, y otro grupo de hospitales desafortunadamente no menor, que difícilmente estará en condiciones para el 2009, lo que obligará una eventual modificación legal para extender el plazo a dicho grupo. Esto último parece totalmente aconsejable, ya que el incluir hospitales por el "sólo ministerio de la ley" que tengan una brecha muy importante pondría en riesgo los objetivos globales de todo el proceso; 2) que persiste una falla de conducción en los establecimientos, prueba de ello es la dificultad para llenar los cargos de directivos por el Sistema de Alta Gerencia y la aún alta rotación de directivos. Sin duda que sin liderazgo efectivo que conduzcan procesos persistentes no hay generación de condiciones para EAR. Dicho de otro modo, la ausencia de liderazgo puede señalarse como la primera y principal amenaza al proceso. Es de esperar que la reciente modificación legal que permite a los directores mantener actividad clínica venga a paliar este grave problema; 3) que los actores aún no han asumido cabalmente sus nuevo roles, prueba de ello es el incipiente avance en generar "carteras de servicio" alineadas hacia el cambio de 
modelo y al insuficiente avance en "contratos" efectivos entre la red y los EAR; 4) que no se han generado los incentivos financieros adecuados, prueba de ello son las dificultades que en la práctica persisten para sostener el presupuesto negociado con los EAR.

Sin duda, que los principales desafíos serán el que los establecimientos alcancen dicha categoría una vez logrados los estándares requeridos, y que las nuevas facultades efectivamente sean útiles para las mejoras en el servicio para lo cual fueron diseñados. Las dificultades observadas son propias de un proceso en marcha de gran envergadura. Estamos a tiempo para identificar obstáculos y proponer con audacia soluciones, reconociendo que en todo proceso de cambios necesariamente hay aciertos y errores. Lo que sin duda no puede haber, es incapacidad para aprender y falta de coraje para asumir los desafíos pendientes.

\section{Referencias}

1.- Artaza O, Martínez F, Sacoto F, Torres-Goitia J, Yáñez L, Yglesias A: Reformas y Financiamiento de Sistemas de Salud de la Subregión Andina. Organismo Andino de Salud Lima Perú, 2004.
2.- Fondo Nacional de Salud: Una mirada a fondo a la modernización del FONASA, 1999.

3.- Fondo Nacional de Salud: FONASA: ¿Una reforma en marcha? Santiago, 1999.

4.- Artaza O, Castillo R, Fábrega R, Quinteros M: Gestión del Cambio: Presentación de dos casos chilenos. Gestión Hospitalaria 4 ${ }^{a}$ Edición Editorial mcgraw-Hill Madrid 2007, págs. 519-29.

5.- Artaza O, Montt J, Vásquez C: Estructura y Recursos Humanos en un Hospital Público Chileno: una experiencia de cambio. Revista Panamericana de la Salud Pública OPS 1997; 5: 342-51.

6.- Artaza O y cols: ¿Cómo preparar a una organización pública para funcionar en un modelo de "Autogestión y satisfacción usuaria?". Cuadernos Médico Sociales XXXVIII, 3, 1997/28-37. Chile.

7.- Artaza $O$ y cols: La calidad y la satisfacción usuaria, pilares estratégicos del cambio. Cuadernos Médico Sociales XXXVIII, 3, 1997/41-47. Chile.

8.- Artaza $O$ y cols: Buscando la Excelencia: Autogestión Clínica en la Atención Hospitalaria. Cuadernos Médico Sociales XXXVIII, 1997/54-59. Chile.

9.- Castillo R, Quinteros M, Ibáñez A: El Hospital Público en Chile, Tomo II "Centros de Responsabilidad". Ministerio de Salud. Chile, 2004.

10.- Serie Cuadernos $N^{o} 1$ : Modelo de Atención Integral de Salud. Ministerio de Salud de Chile, Marzo de 2006.

11.- Ley 19.93: Ley de Autoridad Sanitaria que Modifica D.L. No. 2.763.

12.- Subsecretaría de Salud Pública y Subsecretaría de Redes Asistenciales.

13.- Documento Modelo de Gestión Hospitalario Subsecretaria de Redes Asistenciales distribuido el año 2006. 